\title{
Effect of substrate morphology on stress-tested GaN-on-GaN vertical p-n diodes
}

Prudhvi Ram peri ${ }^{1}$, Kai Fu ${ }^{2}$, Houqiang $\mathrm{Fu}^{3}$, Yuji Zhao ${ }^{3}$ and David J Smith ${ }^{4}$

${ }^{1}$ Arizona State University, tempe, Arizona, United States, 2 arizona state university, United States, ${ }^{3}$ Arizona State University, United States, ${ }^{4}$ Arizona State University, 85281, Arizona, United States

Wide bandgap semiconductors, particularly gallium-nitride $(\mathrm{GaN})$ based devices, are of great interest for high-power electronics because of superior material properties such as wide bandgap (3.44 eV), high thermal conductivity $(\sim 1.5 \mathrm{x} \mathrm{Si})$, high critical electric field $(\sim 10 \mathrm{x} \mathrm{Si})$, and high saturation velocity $(\sim 3 \mathrm{x}$ $\mathrm{Si}$ ). Developments in growth techniques such as hydride vapor pressure epitaxy (HVPE) and ammonothermal methods, have led to the availability of freestanding GaN substrates with defect densities lower than $\sim 106 \mathrm{~cm}-2$, which can then be used to grow epitaxial GaN-on-GaN layers with reduced defect density $(<104 \mathrm{~cm}-2)$. Despite these advances, the random presence of defects is still liable to cause degraded device performance and unreliable device behavior [1-2]. This work has investigated stresstesting of GaN-on-GaN vertical devices that were grown on HVPE substrates from two different sources and one ammonothermal substrate. An unintentionally-doped (UID) GaN drift layer with a thickness range of 2-2.4 microns was grown by metal-organic chemical vapor deposition, followed by overgrowth with Mg-doped p-GaN layers with thicknesses of 300-500 nm. The devices were studied using X-ray topography (XRT), SEM and TEM, and the substrate morphology was correlated with the electrical stresstest results. Samples suitable for cross-sectional TEM observation were prepared by FIB milling using a FEI NOVA 200 dual-beam system, with initial thinning done at $30 \mathrm{keV}$ and final thinning done at $5 \mathrm{keV}$. Scanning electron micrographs were also recorded with the FEI NOVA 200 during progressive milling. A Philips-FEI CM-200 FEG transmission electron microscope (TEM) operated at $200 \mathrm{keV}$ was used for imaging.

Figure 1 shows the XRT image of GaN grown on HVPE substrate-1. In figure 1(a), the XRT image shows a two-dimensional array of dark spots that are roughly equally spaced $(\sim 1 \mathrm{~mm})$. The exact locations of devices fabricated on the wafer are indicated by circles, where white circles indicate devices with reliable (acceptable) performance (Breakdown voltages $\sim 300 \mathrm{~V}$ ) and red circles indicate failed devices (Breakdown voltages $\sim 50-150 \mathrm{~V}$ ). Figure 1(b) shows the plan-view SEM image of the fabricated devices. It is apparent that devices that overlap with the dark spots failed prematurely under stress-testing with very high leakage current ( $>10-6$ A), whereas devices away from the dark spots performed much better. Figure 2 (a) shows the low-magnification plan-view SEM image taken at one dark spot (black circle in insert in Fig. 2a). Figure 2(b) is a medium-magnification SEM image focused on a location indicated by black square in Fig. 2(a) and shows the presence of a large number of defect and surface pits. SEM images taken away from such dark spots showed no visible defects. Figure 2(c)-2(f) shows high-magnification SEM images of defects marked as L1, L2, L3 and L4 in Fig. 2(b). These images show large, deep pits with inverted-hexagonal shape. Some defects are clustered together, and overlapping, to form large pits. Further studies of GaN-on-GaN devices grown on HVPE substrate-2 and ammonothermal substrate are ongoing.

Acknowledgments: This work was supported by ARPA-E Award DE-AR0000868. The authors acknowledge the use of facilities within the John M. Cowley Center for High Resolution Electron Microscopy at Arizona State University. 

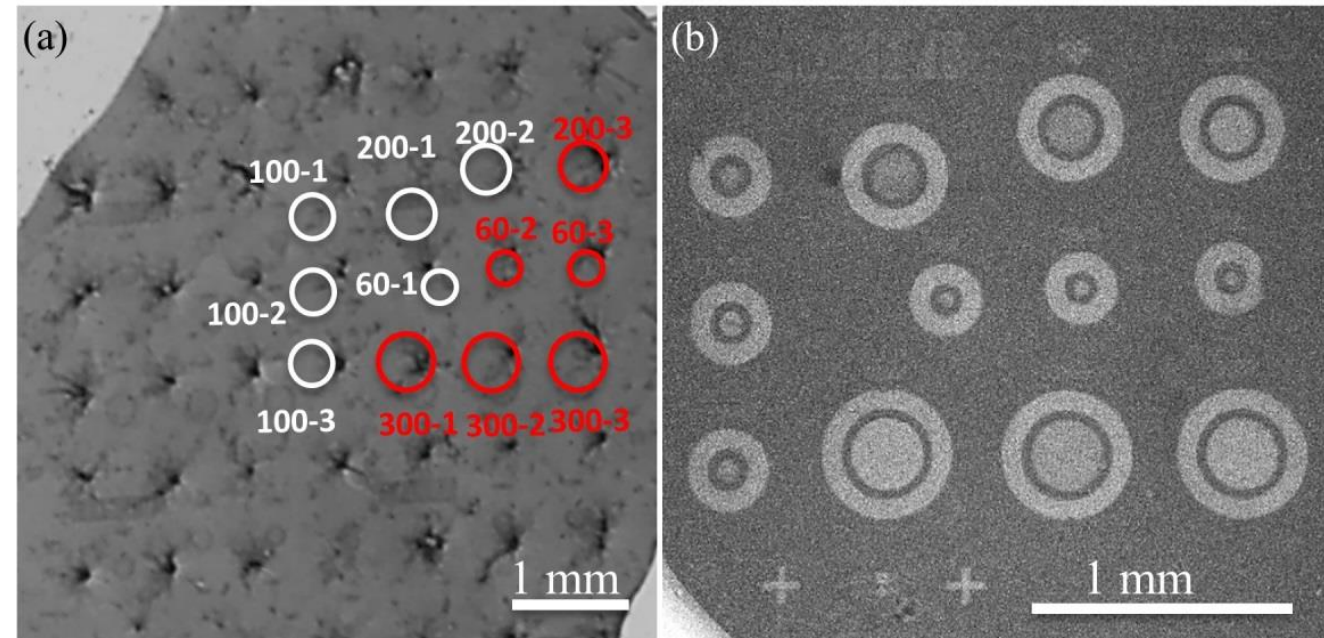

Figure 1. (a) XRT image of GaN-on-GaN substrate-1 showing array of dark-spots. White and red circles indicate locations of devices with good and bad behavior, respectively, while numbers are used to identify devices for stress-testing. (b) SEM image of fabricated devices.
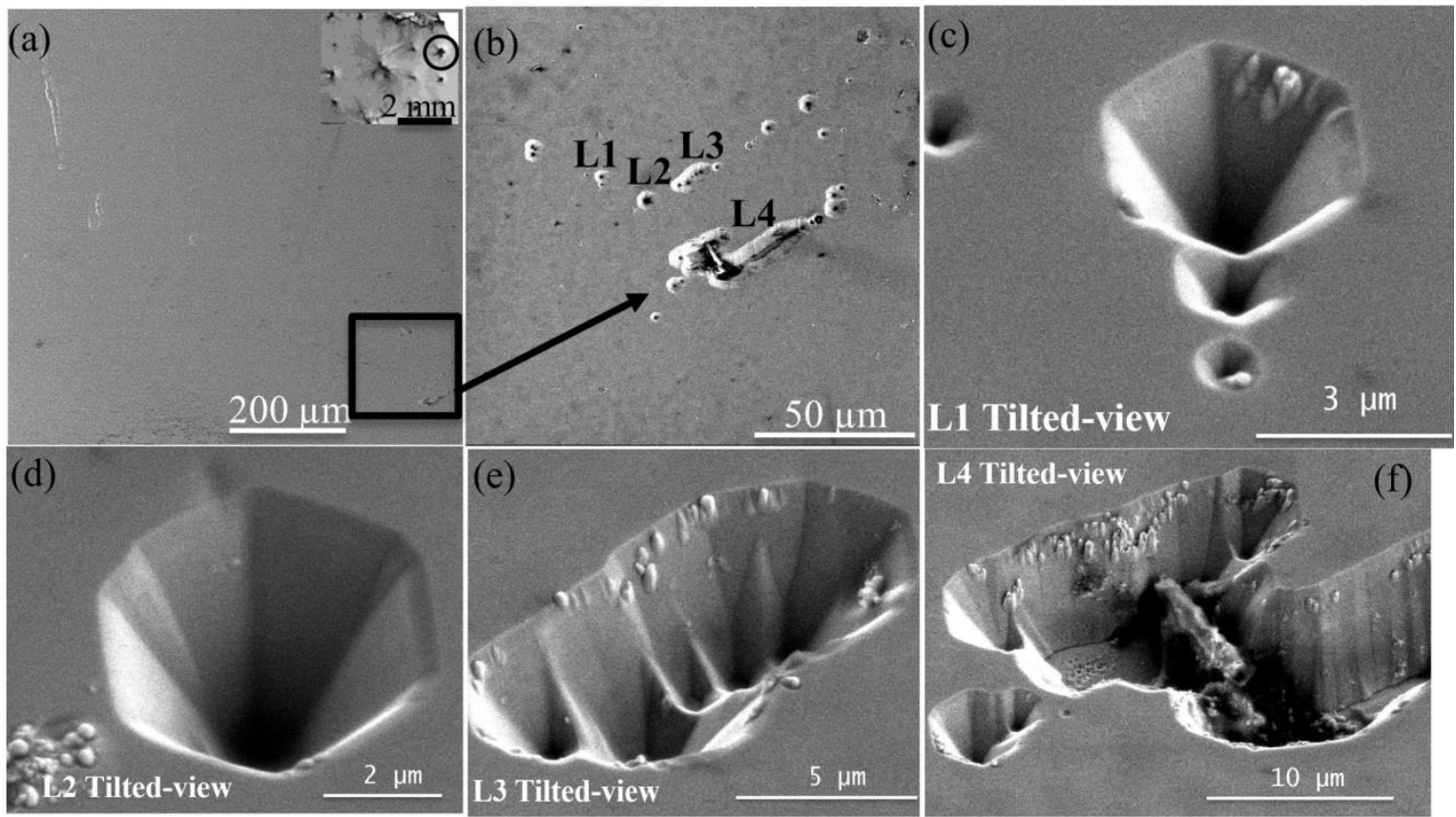

Figure 2. Figure. 2 (a) Low-magnification SEM image taken at a dark-spot (circled), as indicated in XRT image (inset at top right); (b) SEM image from boxed location marked in (a) showing defects; (c) Higher magnification SEM image taken at location L1 marked in (b) shows inverted-pyramid defects; (d) Location L2 shows individual defect, (e) Location L3 shows cluster of defects, and (f) L4 shows cluster of defects coalesced into a macro-scale pit.

\section{References}

[1]. P. Peri, K. Fu, H. Fu, Y. Zhao, and D. J. Smith, J. Electron. Mater. (2021).

[2]. P. Peri, K. Fu, H. Fu, Y. Zhao, and D. J. Smith, J. Vac. Sci. Technol. A 38, 063402 (2020). 